\title{
The source of the solar oscillations: Convective or magnetic?
}

\author{
P. F. Moretti ${ }^{1}$, A. Cacciani ${ }^{2}$, A. Hanslmeier ${ }^{3}$, M. Messerotti $^{4}$, M. Oliviero ${ }^{1}$, W. Otruba ${ }^{5}$, \\ G. Severino ${ }^{1}$, and A. Warmuth ${ }^{3}$
}

1 Osservatorio Astronomico di Capodimonte, Via Moiarello 16, 80131 Napoli, Italy

2 Department of Physics University of Rome, P.le A. Moro 2, 00185 Rome, Italy

3 Institute of Geophysics, Astrophysics and Meteorology of the University of Graz, Universitaetsplatz 5, 8010 Graz, Austria

4 Osservatorio Astronomico di Trieste, Via G.B. Tiepolo 11, 34131 Trieste, Italy

5 Kanzelhöhe Solar Observatory, 9521 Treffen, Austria

Received 14 February 2001 / Accepted 10 April 2001

\begin{abstract}
The origin of solar oscillations has not yet been clearly determined. The downflows due to convective rapid cooling at the surface have been invoked as a possible source.

In this paper we investigate the properties of the source as inferred from the local analysis of the intensity-velocity phase differences. The same spatial and temporal characteristics of other observed events and their correlation with the $\mathrm{H} \alpha$ bright points suggests downward plasma jets related to explosive chromospheric evaporation to be another possible candidate.
\end{abstract}

Key words. sun: oscillations - flares - chromosphere

\section{Introduction}

The five-minute solar oscillations have proved to be a powerful tool in aiding our understanding of the solar interior. The mysterious nature of these oscillations was identified by way of theoretical arguments in 1970 and confirmed by observations in 1975. The oscillations we see on the surface are due to sound waves generated and trapped inside the sun. Sound waves, produced by pressure fluctuations, set the sun vibrating in millions of different patterns or $\mathrm{p}$ modes. In the same way that geologists use seismic waves from earthquakes, helioseismology permits us to deduce the properties of the inner layers of the sun from the study of a perturbation on its surface. Despite the large impact helioseismology has produced in solar physics, the origin of the oscillations has not yet been clearly revealed, that is, the precise mechanism generating the pressure fluctuations is still uncertain. Although simulations seem to sufficiently describe the driving of the p-modes (Stein \& Nordlund 2001), no observational evidence can clearly select this phenomenon as dominant in the excitation.

A first identikit of the source has been drawn by the asymmetry of the p-mode line profiles. If the p-modes are excited as randomly forced, damped harmonic oscillators, the shape of their lines in the power spectra would show a lorentzian profile. The asymmetry in these profiles (Duvall et al. 1993) was originally interpreted as the presence of

Send offprint requests to: P. F. Moretti,

e-mail: moretti@na.astro.it a localised excitation source (Gabriel 1992; Roxburgh \& Vorontsov 1993) in a thin layer near the top of the convection zone.

The presence of a solar "background" in the $\ell-\nu$ diagram of the phase difference between the intensity and velocity signals (I-V), first discovered from ground observations by Deubner et al. (1990) as a negative phase "plateau" and an inter-ridge regime at high- $\ell$ values and low frequency, has been associated with a possible signature of the source of the resonant oscillations.

Different scenarios have been invoked to explain the observed background. The model proposed by Deubner et al. (1996) interprets the background's characteristics in the phase spectra in terms of the response of a cavity in the atmosphere. The photospheric wave field, characterised by evanescent five-minute p-modes and high frequency running acoustic waves, gradually transforms into resonant oscillations, possibly with several eigenfrequencies. The background has been also explained in terms of the acoustic source, primarily identified with the fast cooling associated with the convective "downdrafts" (Skartlien \& Rast 2000). This latter interpretation is consistent with the observations of Goode et al. (1998), Espagnet et al. (1996) and Strous et al. (2000). In fact, seismic events have been detected following a darkening and a collapse of plasma localised in the intergranular lanes. These events last a few minutes and extend over an area of few arcseconds. These downdrafts are thought to be due to buoyant 
acceleration after a radiative cooling at the surface, that is, statistically triggered by convection.

Recently, a correlation between magnetic oscillations, $\mathrm{H} \alpha$ bright points and the background locations (as revealed using a local analysis technique to filter the data) has been found (Moretti et al. 2000). Nevertheless, no direct correlation between the magnetic flux and the downflows has been found so far.

Observational results are often in disagreement when finding spatial and temporal correlation between $\mathrm{Ca} \mathrm{K}$ bright points, magnetic field cancellations, UV jets etc. (Cook et al. 1996; Hoekzema et al. 1997; Lites et al. 1999). Transition region explosive events have been correlated with magnetic cancellations as a consequence of the upward expansion of relaxed magnetic ropes (Chae et al. 1998), but these cancellations are difficult to localise due to their small scales $\left(\simeq 1^{\prime \prime}\right)$.

The evidence in the photosphere of a strong seismic downplume associated with a big flare was detected by MDI (Kosovichev \& Zarkova 1998); and high $\ell$-degree mode excitation has been reported, too (Haber et al. 1988). Similar events were predicted and noted as a possible origin of free oscillations in the sun (Wolff 1972).

In this paper we investigate the possible nature of the source of the solar oscillations when they are identified by the I-V phase differences using a local analysis. The spatial and temporal characteristics of the source locations mimic those of the events related to the explosive chromospheric evaporation (Briand, private communication; Canfield \& Metcalf 1987).

We invoke the downward plumes to relax their energy in the photosphere and trigger seismic events.

\section{The solar background as a signature of the source of the solar oscillations}

\subsection{The $I-V$ phase difference}

The negative phase plateau, first discovered by Deubner et al. (1990), was confirmed by MDI data (Straus et al. 1998). Successively, using GONG data (Oliviero et al. 1999), the negative phase regime has been shown to extend to intermediate and low- $\ell$ values and, at high frequencies, positive values greater than those shown at the p-modes frequencies, have been found. Many of the differences in the values obtained from different data sets (GONG, MDI, VAMOS) can be attributed to different formation heights of the solar lines used and to different $\ell$ and $\nu$ resolution in the I-V phase difference spectra. To date, the experimental results have the following traits:

1 ) the phase values are approximately independent of the degree $\ell$;

2) the phases on the p-mode ridges depend on the height in the solar atmosphere;

3) the phases in the solar background show a step-like behaviour with negative values below about $3.3 \mathrm{mHz}$ and positive values above about $4 \mathrm{mHz}$.

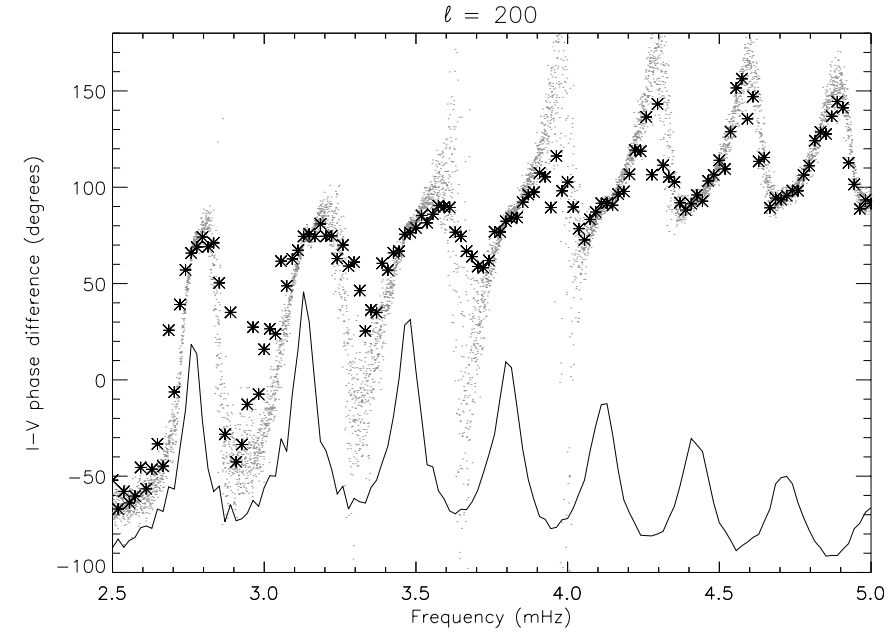

Fig. 1. The I-V phase difference from the analysis of the GONG data in the Ni $676.8 \mathrm{~nm}$ solar line: stars for 15 hour, points for 1 month time-series. The velocity power of the shorter time-series is plotted (solid line) to show the coincidence of the measures only in the high power peaks.

The value of the phase strongly depends on the spherical degree and frequency resolutions. As an example, we show in Figs. 1 and 2 the frequency dependence of the I-V phase difference for the $\mathrm{Ni} 676.8 \mathrm{~nm}$ data (36 days from GONG) and for the $\mathrm{Na} \mathrm{D}$ lines (256 min from VAMOS and Kanzelhöhe, Moretti et al. 1997; Severino et al. 2001; Cacciani et al. 1999).

The measured phase values are related to the ability to distinguish between different phenomena: the resolution in $\ell$ (leakage) or in $\nu$ produces a mixing of the phases weighted by the different powers (Figs. 1 and 2 of Oliviero et al. 1998).

\subsection{The local analysis}

The background has been usually studied via its behaviour in the $\ell-\nu$ diagram: that is, the contribution of the signal filtered by the spherical harmonics is displayed as a function of the spatial scale and of the frequency. In the case of the p-modes, this representation shows the global resonance of the acoustic waves. In this framework, if the source of the oscillations is local and its scale outside the observed $\ell$ range (Skartlien \& Rast 2000), any characteristic of the source should be rather independent of the spatial scales when analysed in a $\ell-\nu$ diagnostic. This is in fact the case in recent results on the phase difference between the intensity and velocity signals previously described. Therefore, it seems reasonable to use another technique to filter the data in order to enhance the local characteristics of the source of the solar oscillations. When the velocity and intensity oscillations are treated locally, they may be different due to mixing with local phenomena: their characteristic spatial distributions on the disk are not washed out by a filter, as the spherical harmonics decomposition. 

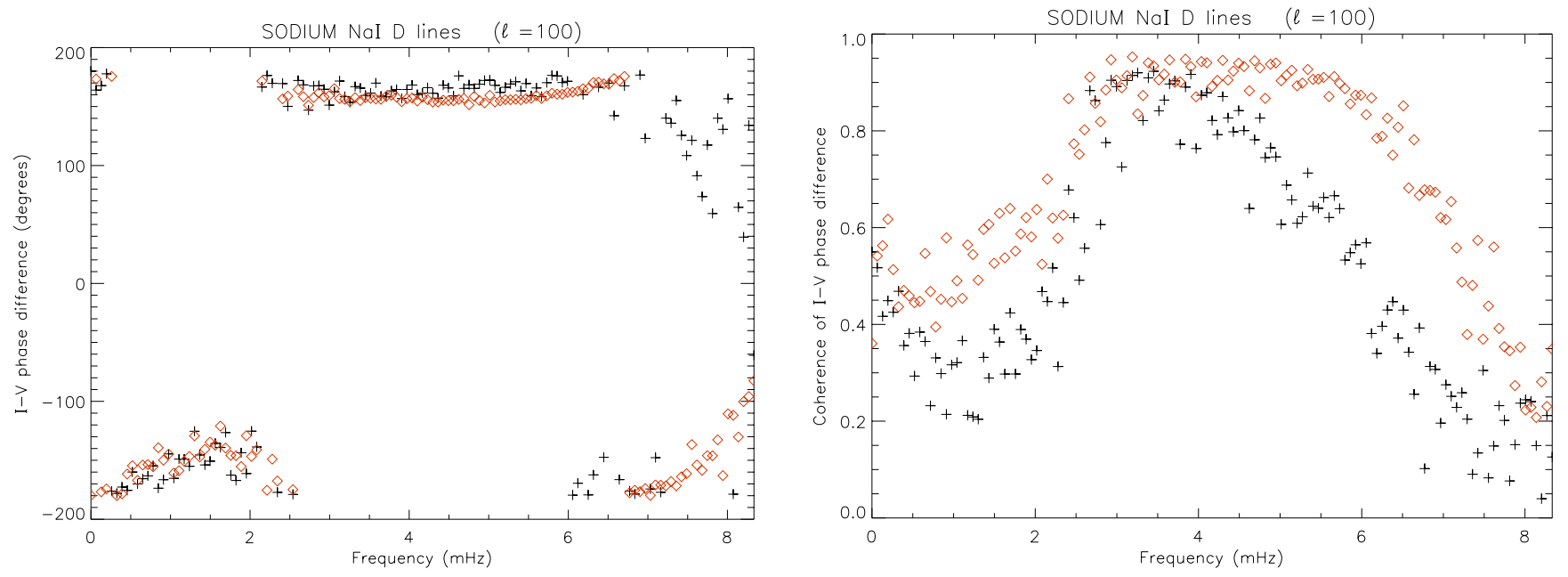

Fig. 2. For $\ell=100$, the I-V phase difference (left) and coherence (right) for the VAMOS (crosses) and Kanzelhöhe (diamonds) data. Both data refer to the $\mathrm{Na} \mathrm{D}$ lines and were obtained from the analysis of $256 \mathrm{~min}$ time-series obtained through a MagnetoOptical Filter.

The local analysis uses a three-dimensional representation, as the distribution on the disk (in $x, y$ ) has to be visualised as a function of the frequency (Lites et al. 1993). In fact, the time-series of the images are pixel-by-pixel fast fourier transformed (FFT) and the power and phase difference maps are obtained. Long time-series will produce a spatial average because of solar rotation and the evolution of structures on the solar surface. The granules and supergranules have time-scales of 10 minutes and hours with scales of $\simeq 1^{\prime \prime}$ and $\simeq 50^{\prime \prime}$ respectively. The structures would lose their identity after a lifetime and a compromise has to be reached, so as not to remove anything but the rotation. As a consequence, the temporal resolution we get is limited by an observing run of a few hours. Nevertheless, it is useful to perform this analysis since it permits us to investigate the spatial distribution of the oscillatory power and its relation to local phenomena. Moreover, in order to evaluate the contributions of the different phenomena to the signal, it is useful to produce the $\ell-\nu$ diagram to make the distinction between the oscillations and the convection easier. With our resolution, the convection contribution is negligible in comparison to that of the p-modes in the five-minute frequency range.

\subsection{The data}

The data consist of three sets of dopplergrams, magnetograms and intensity images obtained with a sodium Magneto-Optical Filter (MOF) at Kanzelhöhe (Cacciani et al. 1999). The images were acquired every minute and $256 \mathrm{~min}$ from each day were selected for the analysis. Some days were analysed and we show the results relative to 30 January 1998. The spatial resolution is $4.3^{\prime \prime} /$ pix. The dopplergrams were calibrated as described in Moretti \& the MOF Development Group 2000; all the images were registered but no correction for the rotation has been applied. The full-disk data were treated locally (pixelby-pixel) and globally (using the spherical harmonics

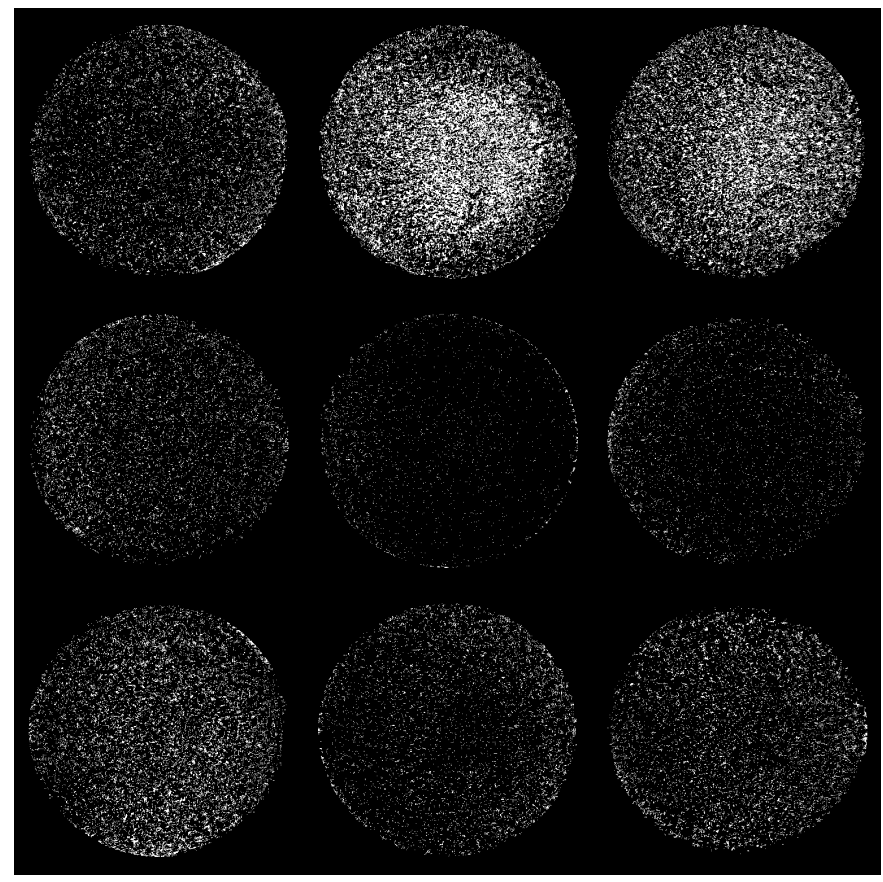

Fig. 3. Examples of the phase maps for, from top to bottom, $+108^{\circ}, 0^{\circ}$ and $-144^{\circ}, \pm 18^{\circ}$ each. From left to right: at 1.3 , $3.3,5.2 \mathrm{mHz}$.

decomposition developed for the VAMOS project, Oliviero et al. 1998) to obtain the power distribution on the disk and the $\ell-\nu$ diagrams. The daily trend was removed in the pixel-by-pixel time-series using a polynomial fit, while a differential filter was used in the spherical harmonics decomposition. The final spectra were corrected for the filtering of the data. Since the dopplergrams were not simultaneous to the intensity images, the correction for the time delay in the phase maps has been introduced. 


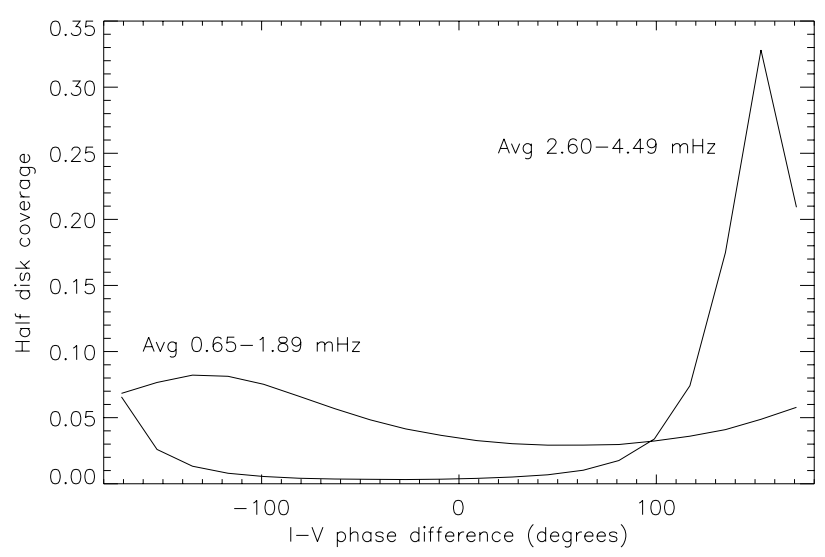

Fig. 4. The probabilities of finding the $\mathrm{I}-\mathrm{V}$ phase values have been averaged in the low frequency (between 0.65 and $1.89 \mathrm{mHz}$ ) and five-minute (between 2.60 and $4.49 \mathrm{mHz}$ ) bands. Two populations are visible in the local analysis as well as in the $\ell-\nu$ diagram (see also Fig. 2). We associate the population with the positive value to the p-modes, and the negative to the solar background.

\subsection{The I-V phase difference when obtained through a narrow passband filter}

A further correction in the phase difference values has to be applied when a narrow passband filter (like a MOF) is used (Moretti et al., to be submitted). In these systems, a velocity shift of the line profile induces an intensity fluctuation as the main undesirable effect. The contamination is larger where the velocity power is stronger and depends on the sensitivity of the signal to the velocity. That is, the contamination mainly changes along the solar disk according to the relative velocity to the Earth. The crosstalk has been modelled for this system and the correction to the phases applied. We note that the error in the corrected phase values may be large but, in this context, does not affect the discussion based on the spatial properties of the I-V phase difference. In fact, the phases' changes are well below the phase difference between the different phenomena.

\section{The analysis and the results}

The phase difference maps have been obtained up to $8.33 \mathrm{mHz}$ (see examples in Fig. 3). The $360^{\circ}$ range was divided into many intervals. The coverage of the phase values at any frequency was computed for an area out to 0.5 radii, and correspond to the probability of finding that phase in the part of the disk where the p-mode interference pattern dominates. In order to enhance the existence of different populations of phase values, an average of the "coverages" was performed for the low frequency and fiveminute bands respectively (Fig. 4). Two populations of phase values are significant on the disk: a positive (around $+140^{\circ}$ ) component and a negative (hereafter called $-144^{\circ}$ or background value), whose value we attribute to the solar background at the sodium D line formation layer (see also Fig. 2).
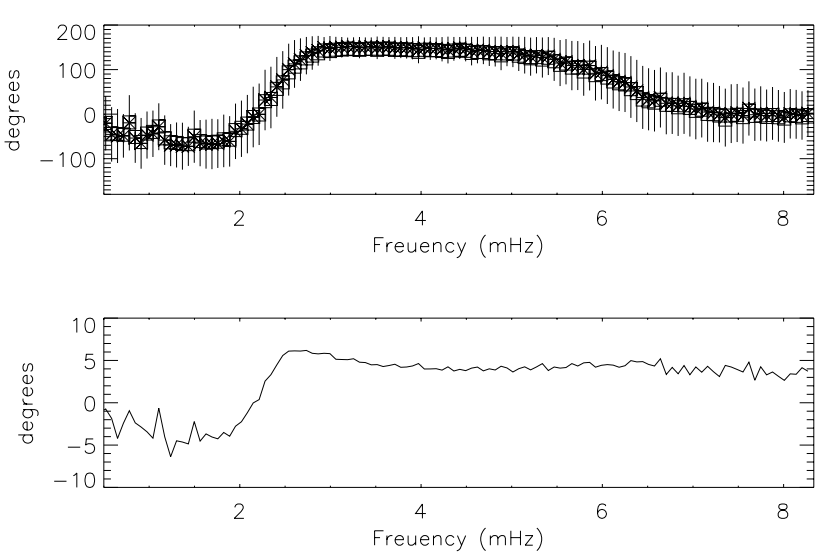

Fig. 5. Top: the I-V phase differences (before crosstalk correction) corresponding to the high velocity power locations out to $0.5 \mathrm{R}$ have been averaged at any frequency. The error bars are the sigma obtained from the spread over the half disk. Bottom: the difference between the values before and after crosstalk correction (Moretti et al., in preparation).

In the sodium results the $-144^{\circ}$ value, clearly attributed to the background in the $\ell-\nu$ diagram at the low frequency band, is visible with the local analysis in the five-minute band too, but not in the $\ell-\nu$ diagram where the low temporal resolution does not permit us to distinguish the low power inter-ridges. On the contrary, in the local analysis, the low power sites are practically localised at any frequency and the $-144^{\circ}$ value can be seen.

In practice, what the spatial recognition performs in the local analysis, the high frequency resolution does in the $\ell-\nu$ diagram. The phase values for the modes have been selected as those corresponding to the locations where the velocity power is greater than a threshold. The threshold was chosen as the mean velocity amplitude computed from the power maps: it changes approximately from $1 \mathrm{~m} / \mathrm{s}$ at low frequencies to $15 \mathrm{~m} / \mathrm{s}$ in the five-minute band. The values for the phase at these locations have been successively averaged over the solar disk and their distribution is shown in Fig. 5.

The phase value corresponding to the high power locations, averaged between 3.2 and $5.2 \mathrm{mHz}$, is $+135^{\circ} \pm 20^{\circ}$. The high power locations in the five-minute band are usually considered to be the places where the interference of the oscillations act constructively, and for this reason we also attribute these values to the "modes" also in the local analysis.

While other phase values seem to be randomly distributed on the disk, at disk center in the five-minute band, the $-144^{\circ}$ value occurs where the velocity power is low and in the low frequency range. In order to make this behaviour visible, the probabilities as previously described have been computed for the locations at different velocity thresholds (see Fig. 6). It is clear that the positive values are the mode values and the power acts as a selective filter for the $-144^{\circ}$ value. The $-144^{\circ}$ is well visible at low frequencies, where no powerful phenomena 

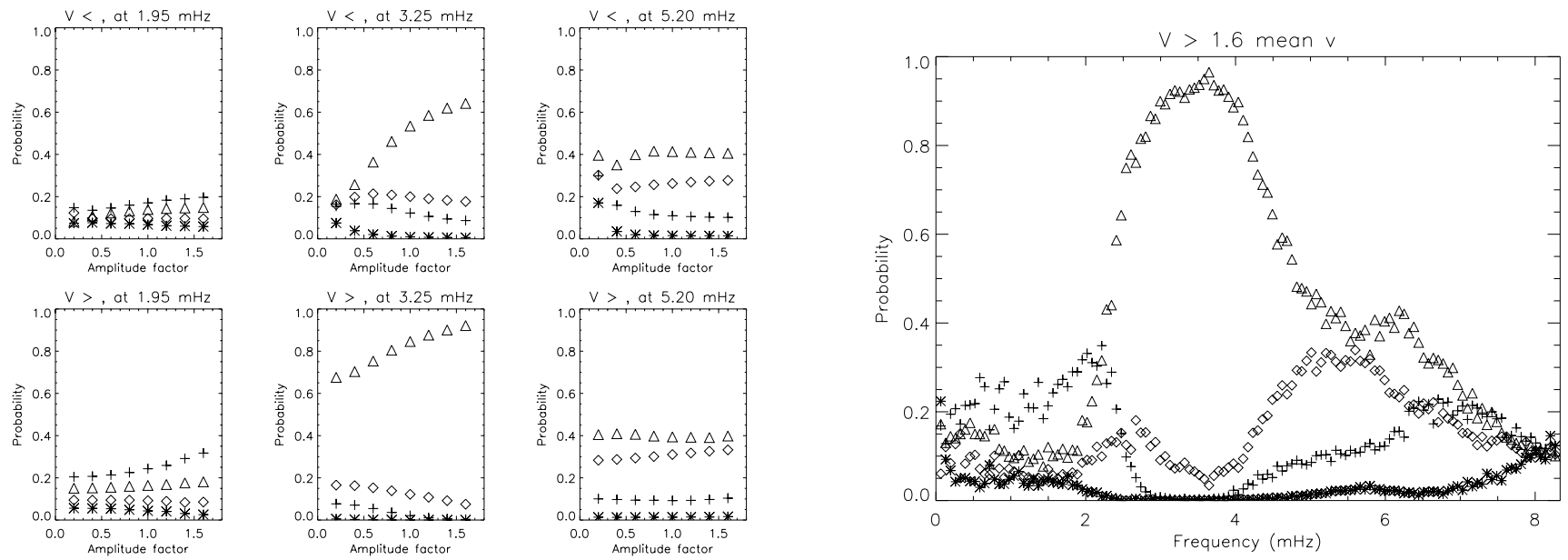

Fig. 6. Left panels: the probability of finding a phase value (within $\pm 18^{\circ}$ ) computed at the locations where the velocity amplitude is lower (top) or greater (bottom) than a threshold (see text) is shown at three frequency samples. The $x$-axis represents the factor by which the threshold has been multiplied. Crosses, stars, diamonds and triangles represent $-144^{\circ}, 0^{\circ},+108^{\circ},+126^{\circ}$ respectively. The velocity power acts as a selective filter at the five-minute band. Right panel: The frequency dependence of the phase probabilities at the high power locations (crosses, stars, diamonds and triangles for $-144^{\circ}, 0^{\circ},+108^{\circ},+126^{\circ}$ respectively $\left( \pm 18^{\circ}\right)$, multiplying factor $\left.=1.6\right)$.

dominate the disk. The frequency dependence of the phase difference is shown in Fig. 5 for the powerful locations: the five and three-minute bands show slightly different values. This difference, not visible in the $\ell-\nu$ diagram, can be attributed to a different dilution of the phenomena when they are spatially averaged. In fact, since the velocity amplitude in the three-minute band is small, a selection of the modes cannot be performed as clearly as in the fiveminute band. As a result, the higher value of the p-modes phase is lowered (this effect could also justify the results in Khomenko 2001).

Even if the spatial resolution of the data does not permit us to distinguish unequivocally the magnetic network, a correlation with the magnetic field and its oscillations has been performed using a simultaneous data set of longitudinal magnetograms. The results do not show a oneto-one correspondence between the magnetic field and the $-144^{\circ}$ value, but this latter value is often shown in the magnetic locations, where the five-minute power is usually reduced (Fig. 7).

When a correlation is performed between the maps of the $-144^{\circ}$ value averaged in the low frequency band and the magnetic power maps, the correspondence to the $H \alpha$ bright points is visible (Fig. 8, see also Moretti et al. 2000). The morphology is well recognisable, even if the structures are not co-spatial (due to the spread of the magnetic lines, due to the non-vertical propagation of the jets etc.). Nevertheless, this is not a proof of a physical relation between the phenomena at different layers (we note that the background is also localised where the magnetic power is larger, and that the magnetic signatures are typically related to the $H \alpha$ bright points).

Is the velocity power really acting as a filter on the visibility of the background value whose distribution is indeed intrinsically uniform on the disk? If this is the case,

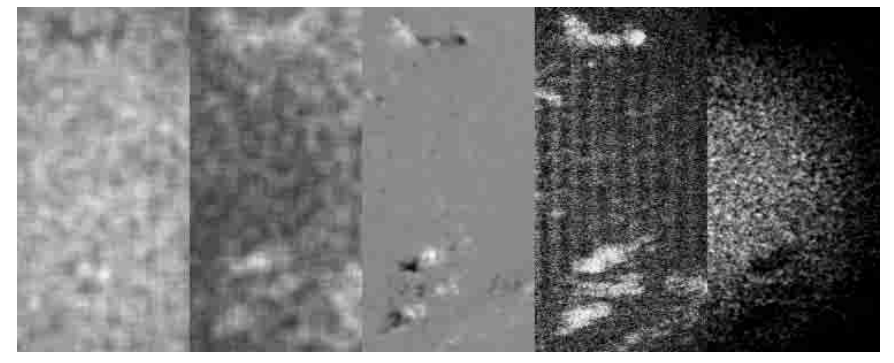

Fig. 7. From left to right: the $-144^{\circ}$ phase difference probabilities averaged between 1.6 and $2.6 \mathrm{mHz}$ (white means high probability), the $-144^{\circ}$ phase difference probabilities averaged between 3.3 and $4.3 \mathrm{mHz}$, a longitudinal magnetogram, the magnetic power map at $3.3 \mathrm{mHz}$, the velocity power map at $3.3 \mathrm{mHz}$. The vertical size is approximately 0.75 solar radii.

we expect an increase in its coverage where the p-modes do not dominate, that is, in the low frequency band. In fact, the constructive interference patterns of the p-modes should not hide the background locations.

Since the $360^{\circ}$ phase range has been divided into 10 intervals each $36^{\circ}$ wide, a 0.1 coverage is expected for a uniform distribution. The increase of the background coverage from less than 0.1 in the five-minute band to 0.2 in the low frequency band can be interpreted in two ways, as described in Fig. 9 (see also Fig. 6). From the coverage of the p-mode phase in the five-minute band $(0.9=0.1+$ $\left.\frac{\pi R^{2}}{2 \pi R \mathrm{~d} R+\pi R^{2}}\right)$, the ratio between a cell of the constructive interference pattern and the border lines can be deduced $\left(R / \mathrm{d} R\right.$ is equal to 8 , that is an area of approximately $70^{\prime \prime}$ of diameter if the border is chosen as one pixel wide). As a result, the 0.2 probability to find the $-144^{\circ}$ value in the low frequency band can be produced by a 0.5 non random coverage of the border lines or an uniform 0.2 coverage of the disk. The former case is suggested to be the case. In 

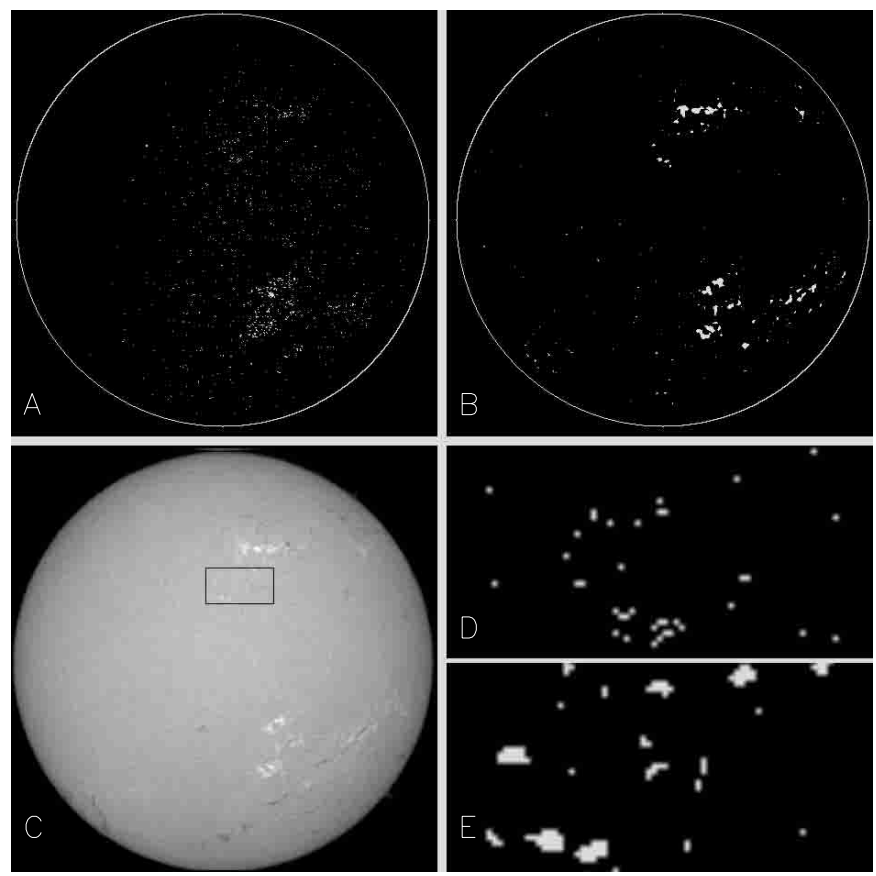

Fig. 8. A) A mask obtained as the product of the strongest magnetic power locations and the phase map at $-144^{\circ}$ at the low frequency band. B) The corresponding $H \alpha$ image (only the very bright points have been selected). C) The raw $H \alpha$ image. The marked area is shown in the next two panels. D) The zoom of the mask in A. E) The zoom of the mask of the $H \alpha$ bright points.

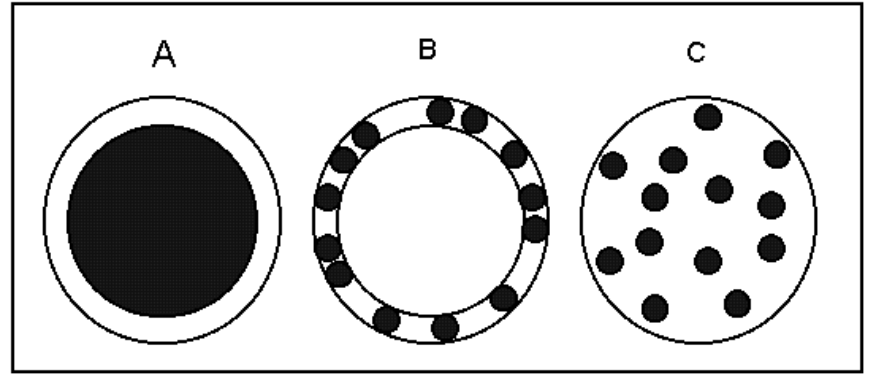

Fig. 9. Let us assume these simplified spatial distributions for the coverages of the p-modes at the five-minute band (A), and for two possible background spatial distributions at low frequencies (B and C). A) The p-modes fill the area of a single cell of the constructive interference pattern (in black) whose radius is denoted by $R . \mathrm{d} R$ is the width of the circular corona representing the border lines separating different cells. From the coverage of the p-modes phase: $0.9=0.1+\frac{\pi R^{2}}{2 \pi R \mathrm{~d} R+\pi R^{2}}$, we obtain $R / \mathrm{d} R=8$. Assuming $\mathrm{d} R=1$ pix, $R \simeq 35^{\prime \prime}$. B) The background is localised in the border line of the cell of the constructive interference pattern of the p-modes. C) The background uniformly covers the disk.

fact, the spatial distributions of the $-144^{\circ}$ maps at low frequencies seems not to be uniform but shows a clustering at scales of the order of $50^{\prime \prime}$ (see Fig. 10).

In addition, we computed the probability of finding a phase value (at the usual ten phase intervals $36^{\circ}$ wide each), in the same pixel, at different frequencies, in the
Table 1. The coverage of points where a phase value $\left( \pm 18^{\circ}\right.$, first column) occurs at least once between 1.6 and $2.6 \mathrm{mHz}$ (top) and 3.2 and $5.2 \mathrm{mHz}$ (bottom) have been computed (second column). Those points whose probability in that frequency interval exceeds 0.333 have been selected and the probability of occurring is shown in the third column. For an uniform distribution, the probability of finding a point with a probability larger than 0.333 is $8 \%$.

\begin{tabular}{ccc}
\hline \multicolumn{3}{c}{ Averaged between 1.6 and 2.6 mHz } \\
\hline phase $\pm 18^{\circ}$ & coverage (\%) & high prob. points (\%) \\
$-162^{\circ}$ & 67.4 & 0.7 \\
$-126^{\circ}$ & 96.1 & 19.9 \\
$-90^{\circ}$ & 87.9 & 4.4 \\
$-54^{\circ}$ & 67.7 & 0.7 \\
$-18^{\circ}$ & 53.5 & 0.2 \\
$+18^{\circ}$ & 51.1 & 0.2 \\
$+54^{\circ}$ & 61.0 & 0.4 \\
$+90^{\circ}$ & 84.9 & 2.9 \\
$+126^{\circ}$ & 97.2 & 27.4 \\
$+162^{\circ}$ & 65.1 & 0.7 \\
\hline \multicolumn{4}{c}{ Averaged between 3.2 and $5.2 \mathrm{mHz}$} \\
\hline phase $\pm 18^{\circ}$ & coverage (\%) & high prob. points $(\%)$ \\
$-162^{\circ}$ & 50.2 & 0.0 \\
$-126^{\circ}$ & 86.1 & 0.0 \\
$-90^{\circ}$ & 54.6 & 0.0 \\
$-54^{\circ}$ & 31.4 & 0.0 \\
$-18^{\circ}$ & 28.6 & 0.0 \\
$+18^{\circ}$ & 38.1 & 0.0 \\
$+54^{\circ}$ & 70.1 & 0.0 \\
$+90^{\circ}$ & 99.8 & 18.3 \\
$+126^{\circ}$ & 100.0 & 98.3 \\
$+162^{\circ}$ & 65.9 & 0.4 \\
\hline
\end{tabular}

low frequency (between 1.6 and $2.6 \mathrm{mHz}$ ) and five-minute (between 3.2 and $5.2 \mathrm{mHz}$ ) bands respectively. This corresponds exactly to the definition of the probability as the number of successes divided by the number of throws, where the throws are in our case the frequencies. The probability for a uniform distribution is 0.1 , since ten intervals have been selected. We counted the number of pixels whose probability exceeds 0.333 (in the case of an uniform distribution, we expect a $8 \%$ of the points to show a probability larger than 0.333 ).

These numbers (shown in Table 1) clearly show that the $-144^{\circ}$ locations are not randomly distributed at any frequency but seem to be associated with a spatially located phenomenon. In fact, since the "throws" are the frequencies, this means that something has occurred in a place with a temporal behaviour whose transform is approximately flat or confined in a $36^{\circ}$ interval at low frequencies. The pixels where the probability of finding the $-144^{\circ}$ value is high over the whole low frequency band have been selected and their time-series compared to those where the p-modes value dominate. Some darkenings, lasting few minutes, at intervals of about $100 \mathrm{~min}$, are visible. These results suggest the presence of localised events, whose detection in the time-series is difficult due to their short duration and to their possible small scales. 


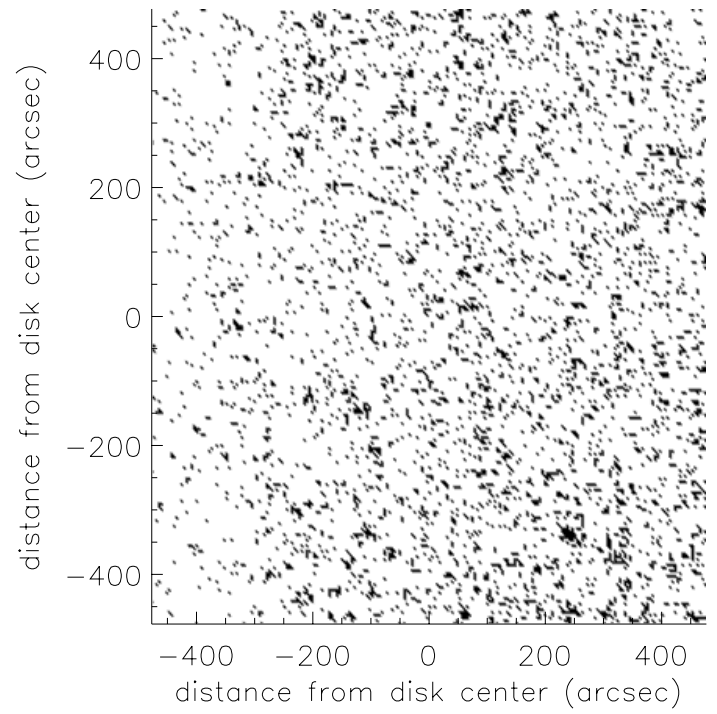

Fig. 10. The probability of finding the $-144^{\circ}$ phase at the low frequency band, in the case of an uniform distribution, is 0.1 (since 10 intervals in phase have been chosen). The pixels where this probability is larger than 0.333 have been selected and shown (in black). The background locations seem to cluster around cells of about $50^{\prime \prime}$.

If the same results were interpreted as the filtering of p-modes on the background produced by the atmospheric cavity, as proposed by Deubner et al. (1996), why do the background locations correspond to the low velocity p-modes power locations in the low frequency range too? Why does the background maintain the characteristic of small signatures clustered in larger structures?

To confirm the hypothesis of the background generated by localised phenomena, the autocorrelation image of the coverage maps at disk center (out to $0.5 \mathrm{R}$ ) at any phase interval has been performed. The east-west and northsouth standard deviations for a 2-D Gaussian fit have been computed. In Fig. 11, their difference is shown versus the frequency. The p-modes locations show the east-west elongation, that is, the associated structures (the constructive interference patterns of the five-minute oscillations, lasting more than the $4 \mathrm{~h}$ duration of the observing run) rotate, while the $-144^{\circ}$ locations, whose typical scale is one pixel, do not. This means that the background locations are related to rotating structures well below the resolution or to structures at the limit of the resolution but lasting a period whose trace during the rotation is confined in one pixel, that is they last less than $30 \mathrm{~min}$.

In the case of the cavity hypothesis, if the interference patterns related to the cavity itself are supposed to last at least $4 \mathrm{~h}$, the results limit their spatial dimension to subarcsec scales.

\section{Discussion}

The I-V phase $\ell-\nu$ diagram in the sodium $\mathrm{D}$ lines shows two dominant values: a positive one on the p-modes and a negative one on the so called "background". The data

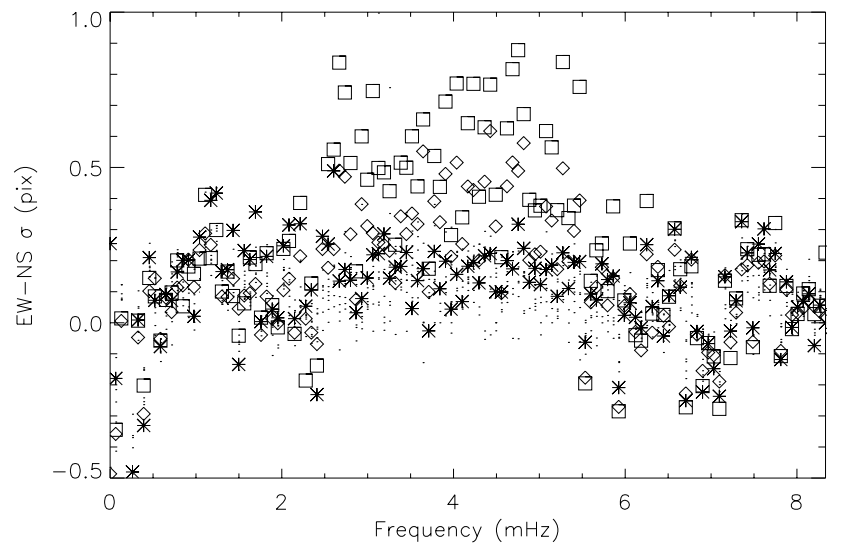

Fig. 11. The east-west and north-south sigmas for a twodimensional Gaussian fit were computed for the autocorrelation image of the coverage maps at disk center (out to $0.5 \mathrm{R}$ ) at any phase interval. The difference between the sigmas along the east-west and north-south directions is plotted. The rotation imprinting is visible in the mode phase at the five minute band. Symbols: squares for $+90^{\circ}$, diamonds for $54^{\circ}$, stars for $-126^{\circ}$, points for the other intervals, $+36^{\circ}$ wide each.

analysed locally show the same I-V phase values in the $\ell-\nu$ diagram.

In the five minute band, the phase found with the local analysis corresponding to the high velocity power locations, is that found in the peaks of the p-modes, while the negative phase is found where the velocity power is low.

As an analogy, we attribute the negative value obtained through the local analysis to the signature of the solar background. We investigated the spatial distribution of the background along the frequency domain in order to infer some characteristics of the source of the solar oscillations.

We summarise the obtained results and a possible interpretation. 1) The background locations, in the fiveminute band, are associated with those points where the velocity power is low. This could mean that the p-modes are acting as a selective filter for a uniform background distribution over the disk. At this point, the correspondence to the magnetic oscillating points is not a proof of a physical relation between the magnetic field and the background, since the magnetic points usually correspond to low velocity power locations. For this reason, the spatial distribution of the background is studied at low frequencies, where the contamination due to p-modes is largely reduced. 2) At low frequencies, the probability of finding the background at the same location is much larger than expected. The slight increase in the area filled by the background is compatible with a 0.5 coverage of $4^{\prime \prime}$ border line of a $70^{\prime \prime}$ diameter region; the non-uniform spatial distribution is confirmed by the behaviour of the coverage, that seems to cluster around structures of the same order of magnitude. This is consistent with the observations of Chae et al. (1998), where a preferential occurrence of the events is reported. This suggests the presence of localised phenomena, whose temporal behaviour reproduce a phase 
difference that is constant in a $36^{\circ}$ interval between 1.6 and $2.6 \mathrm{mHz}$. 3) The autocorrelation of the phase coverage maps shows the rotation of the structures associated with the p-modes (at the five-minute band), but not of the background, whose characteristic scale is of the order of one pixel. This can be interpreted as a rotating subarcsec structure during the observing run, or to structures at the limit of the spatial resolution but lasting a period whose trace during the rotation at disk center is confined in one pixel, that is, less than $30 \mathrm{~min}$.

The delay between the intensity and velocity pulses can be accepted up to $60 \mathrm{~s}$ : this value causes a $20^{\circ}$ linear trend in the phase value in $1 \mathrm{mHz}$ frequency interval and is considered as constant in an analysis performed to search for the co-spatial background events, as we described in Sect. 2 of the discussion.

These characteristics seem to match very well those of the downflows required to generate the background's characteristics in the $\ell-\nu$ diagram (according to the model of Skartlien \& Rast 2000), even if the spatial resolution is low. This limitation could make the determination of the phase values uncertain, but we remark that the starting point of our discussion is that we are dealing with two distinct observed phases. No hypotheses were formulated regarding the phenomena that would reproduce these values, but we attributed them to the p-modes and the background signatures. Some processes are invoked to justify the spatial distribution of the background locations. The one we propose on the basis of some observational evidence is introduced below and discussed in the last section. Some questions remain unanswered. What are the border lines where the background locations seem to cluster? Are they related to the supergranular lanes, the magnetic network, or something else?

Any attempt to correlate the structures usually fails but, nevertheless, many coincidences in the shape are clearly visible (see Fig. 8) with the events associated with the bright points in chromosphere. So, if the "convective" downflows are correlated to the intergranular lanes, where the magnetic cancellations are expected to occur, and if the latter process produces an observed upward plasma jet, this suggests that the photospheric downflows could be related to the downward counterpart.

Some observations confirm the perturbation at the surface induced by a downplume after a big flare (Kosovichev \& Zarchova 1998) and the lack of co-spatiality suggests the non-vertical propagation of the plasma. Is the energy input from these events compatible with the observed balance of the p-modes during the solar cycle? It has been established that the magnetic field reduces the power of the p-modes (Chaplin et al. 2000; Braun \& Lindsey 1999; Komm et al. 2000). Nevertheless, the energy input in the solar oscillations seems to be independent of the cycle (Komm et al. 2000; Chaplin et al. 2000). This evidence attributes a passive role to the magnetic field. On the contrary, stellar observations show a strong correlation between convection and the magnetic activity (Pettersen 1989). In fact, the turbulent motions stress the magnetic field lines resulting in a continuous flaring. The cycle is mainly related to the strong, stable magnetic structures, and the large and visible events in the maxima are rare and negligible in the energy balance. This could invoke two distinct processes coupling the oscillations and the magnetic field: the absorption due to the interaction with the long-term strong magnetic structures and the continuous energy input due the injection of downflowing plasma at small scales, caused by a flaring activity. For this reason, if the background in the I- $\mathrm{V}$ phase difference is believed to be the signature of the source of the solar oscillations, we suggest this source to be "magnetic" instead of "convective", even if the energy source is still of a kinetic nature.

\section{The energy input from the downward jets}

The observational and theoretical results do not reveal a clear picture of the source of the solar oscillations. The asymmetries of the p-mode spectral lines have been interpreted as the signature of a spatially confined source, just below the surface of the p-mode cavity (Duvall et al. 1993). Recently, a model, not based on an explicit "depth dependent" source, explains the same effect on the profiles (Magrí et al. 2001).

The continuous, randomly distributed downflow caused by rapid cooling at the surface is thought to be the trigger of solar oscillations (Goldreich et al. 1994). These downflows have been observed and correlated with seismic events (Goode et al. 1998) and have been used to explain the global behaviour of the solar background in the intensity-velocity phase difference (Skartlien \& Rast 2000). These convective downflows have spatial scales of a few arcseconds and a duration of the order of few minutes.

From the analysis of several years of power spectra, the energy and energy input of the p-modes have been studied through the solar cycle. The most recent results give for the total energy and for the total energy input $4.2 \times 10^{34}$ erg and $1.9 \times 10^{29} \mathrm{erg} \mathrm{s}^{-1}$ respectively (Komm et al. 2000). The former varies with magnetic activity (confirming their absorption) while the latter is compatible with zero changes (Komm et al. 2000; Chaplin et al. 2000). The data analysis of the ongoing maximum will confirm or reject this last conclusion.

In the following, we discuss the downflows associated with the flare occurrence to be a possible source of the solar oscillations.

This hypotheses is suggested by the observational evidence of the impact into the photosphere of the downward plume caused by a big flare (Kosovichev \& Zarchova 1998) and of the recent correlations between the $\mathrm{H} \alpha$ bright points and the locations of the solar background described in this paper.

The UV brightening, the X-ray blue asymmetry in the coronal $\mathrm{Ca}$ lines and the $\mathrm{H} \alpha$ red asymmetry in the bright kernels are associated with the occurrence of flares. The physical mechanisms advanced to explain these phenomena depend on the observed velocities, momentum, energy 
etc. Let us select the explosive chromospheric evaporation model (Fisher et al. 1985a) in order to compute the downward energy flow. Why this process? One reason is that the observed main characteristics of these events seem to reproduce those we want in the local behaviour of the $\mathrm{I}-\mathrm{V}$ phase: they have $\mathrm{H} \alpha$ counterpart, usually reported in the bright points at spatial scales of some arcsec and one minute timescales (Canfield et al. 1987; Canfield \& Metcalf 1987) and they are usually located at the border of the supergranular cells (Henoux 2001).

The leit-motif of this discussion is: several flares are observed, many are related to the sudden creation of a high-pressure region at the footpoint of a coronal loop causing balanced downward-upward plasma jets (Canfield et al. 1987); the downward jets are those observed in the $\mathrm{H} \alpha$ line; the upward are observed in UV and X-rays and are invoked to explain a portion of the coronal heating. We estimate, only from observational evidence, the energy input to the p-modes as computed from the kinetic energy injected by the downward counterparts of the UV jets at scales of approximately 2 arcsec.

There is an energy flux threshold for explosive evaporation: it occurs when the upper chromosphere is unable to radiate the flare energy deposited there. If it occurs, the plasma driven downward (with a maximum velocity of $100 \mathrm{~m} / \mathrm{s}$ ) is cool and dense (Fisher et al. 1985b). For this mechanism, the downward and upward momenta are balanced (and should be seen in the $\mathrm{H} \alpha$ and UV as impulsive events). We estimate the downward energy input using only some conservative values obtained from the observations, that is, we do not include the contribution below the limit of detection of the actual instrumentation.

Following Canfield et al. (1987), the momentum of the downward plume is $2 \times 10^{21} \mathrm{~g} \mathrm{~cm} \mathrm{~s}^{-1}$ and the average velocity $30 \mathrm{Km} \mathrm{s}^{-1}$, that is, a kinetic energy per event equal to $3 \times 10^{27} \mathrm{erg}$. In order to estimate the total injected energy input, the global Sun birthrate is needed. From the related UV measurements, the global Sun birthrate of the impulsive brightenings strongly depends on the threshold used to select the events. Using the recent results from EIT on board SOHO (Berghmans et al. 1998), it can vary from 10 to $40 \mathrm{~s}^{-1}$. In summary, the energy input spans from $\simeq 3.3 \times 10^{28} \mathrm{erg} \mathrm{s}^{-1}$ to $\simeq 1.3 \times 10^{29} \mathrm{erg} \mathrm{s}^{-1}$. This range is consistent with the value measured by Komm et al. (2000). We have assumed that when the $\mathrm{H} \alpha$ and UV jets are observed at the arcsec scales, the energy deposition rate in the chromosphere has been enough to inject the plasma in the lower layers of the solar atmosphere (referred as an "electron beam flare", Mullan 1989). Did those downward jets reach the photosphere? Are they reflected by the magnetic topology or squashed into the higher density layers? The total energy input can be multiplied by a penetration coefficient to take into account these possibilities. Nevertheless, the results obtained by the Kosovichev \& Zarchova show the seismic event to mainly preserve in the photosphere the spatial and temporal characteristics observed in the higher layers. Moreover, signatures at the sodium formation layers have been reported (Warmuth et al. 2000) and are commonly revealed (Moretti et al., in preparation). This suggests the penetration of such jets to be high.

Larger flares have longer temporal and larger spatial scales and should not satisfy the threshold imposed by the energy deposition rate to initiate the explosions. Hence, these events should not be included in any energy balance. Furthermore, the sole energy input estimate is not a selective constraint for the candidates of the source: the involved energy is so small that many processes can easily match the values.

In summary, if we believe that the downward plasma jets produced by the explosive chromospheric evaporation release their energy "somewhere" in the photosphere, the observed energy input for the p-modes can be explained. Moreover, these plumes show the same spatial and temporal characteristics of the events required to explain the solar background as the signature of the source of the oscillations. Last, but not least, the results from a local analysis, even with a low spatial resolution, suggest that the location of the solar background mimics the same events.

We are not claiming the downflows observed in the photosphere to be the "surviving" tails of the jets produced above the surface, but rather, suggest that this plasma rain is a forcing mechanism for the convective instabilities causing the downflows. Higher resolution, multi-wavelength data are needed to confirm a physical connection between the downward plasma injected from the explosive chromospheric events and the photospheric motions underlying the negative intensity-velocity phase difference regime.

Acknowledgements. P.F.M. thanks Federica Brandizzi.

We also thank V. Andretta, E. Busá, B. Cullmann, J. Ireland, S. M. Jefferies, C. Lindsey, Th. Pettauer and Th. Straus.

\section{References}

Braun, D. C., \& Lindsey, C. 1999, ApJ, 513, 79

Berghmans, D., Clette, F., \& Moses, D. 1998, ApJ, 336, 1039

Cacciani, A., Moretti, P. F., Hanslmeier, A., et al. 1999, in Motions in the Solar Atmosphere, ed. A. Hanslmeier, \& M. Messerotti (Kluwer Academic Publishers), 271

Canfield, R. C., Metcalf, T. R., Strong, K. T., \& Zarro, D. M. 1987, Nature, 326, 165

Canfield, R. C., \& Metcalf, T. R. 1987, ApJ, 321, 586

Chae, J., Wang, H., Lee, C., Goode, P. R., \& Schouhle, U. 1998, ApJ, 497, L109

Chaplin, W. J., Elsworth, Y., Isaak, G. R., Miller, B. A., \& New, R. 2000, MNRAS, 313, 32

Cook, J. W., Rutten, R. J., \& Hoekzema, N. M. 1996, ApJ, 470,647

Deubner, F. L., Fleck, B., Marmolino, C., \& Severino, G. 1990, A\&A, 236, 509

Deubner, F. L., Waldschik, Th., \& Steffens, S. 1996, A\&A, 307, 936

Duvall, T. L., Jefferies, S. M., Harvey, J. W., Osaki, Y., \& Pomerantz, M. A. 1993, ApJ, 410, 829

Espagnet, O., Muller, R., Rouder, Th., Mein, N., \& Malherbe, J. M. 1996, A\&A, 313, 297 
Fisher, C. H., Canfield, R. C., \& Clymont, A. N. 1985a, ApJ, 289,425

Fisher, C. H., Canfield, R. C., \& Clymont, A. N. 1985b, ApJ, 289,434

Gabriel, M. 1992, A\&A, 265, 771

Goode, P. R., Strous, L. H., Rimmele, T. R., \& Stebbins, R. T. 1998, ApJ, 495, L27

Goldreich, P., Murray, N., \& Kumar, P. 1994, ApJ, 424, 466

Khomenko, E. V. 2001, in SOHO 10/ GONG 2000 Workshop: Helio- and Asteroseismology at the Dawn of the Millennium, ESA SP-464, 589

Komm, R. W., Howe, R., \& Hill, F. 2000, ApJ, 543, 472

Haber, D. A., Toomre, J. Hill, F., \& Gough, D. 1988, ESA SP-286, 301

Henoux, J. K. 2001, in Themis and the new frontiers of solar atmosphere dynamics, Il Nuovo Cimento B, in press

Hoekzema, N. M., Rutten, R. J., \& Cook, J. W. 1997, ApJ, 474,518

Kosovichev, A. G., \& Zarkova, V. V. 1998, Nature, 393, 317

Lites, B. W., Rutten, R. J., \& Kalkofen, W. 1993, ApJ, 414, L345

Lites, B. W., Rutten, R. J., \& Berger, T. E. 1999, ApJ, 517, L1013

Magrí, M., Oliviero, M., Severino, G., \& Straus, Th. 2001, in SOHO 10/ GONG 2000 Workshop: Helio- and Asteroseismology at the Dawn of the Millennium, ESA SP-464, 653

Mullan, D. J. 1989, Solar Phys., 121, 239

Moretti, P. F., Severino, G., Cauzzi, G., et al. 1997, in
SCORe'96: Solar Convection and Oscillations and their Relationship, ed. F. P. Pijpers, J. Christensen-Dalsgaard, \& C. S. Rosenthal (Kluwer Academic Publishers), Astrophysics and Space Science Library, 225, 293

Moretti, P. F., Cacciani, A., Hanslmeier, A., et al. 2000, in Dynamics of the Sun, ed. A. Hanslmeier, \& M. Messerotti (Kluwer Academic Publishers), in press

Moretti, P. F. 2000, The MOF Development Group, Solar Phys., 196, 51

Oliviero, M., Severino, G., \& Straus, Th. 1998, ESA-SP418, 275

Oliviero, M., Severino, G., Straus, Th., Jefferies, S. M., \& Apporchaux, T. 1999, ApJ, 516, L45

Pettersen, B. R. 1989, Solar Phys., 121, 299

Roxburgh, I. W., \& Vorontsov, S. V. 1995, MNRAS, 272, 850

Severino, G., Moretti, P. F., Oliviero, M., \& The VAMOS Team 2001, in SOHO 10/ GONG 2000 Workshop: Helioand Asteroseismology at the Dawn of the Millennium, ESA SP-464, 337

Skartlien, R., \& Rast, M. P. 2000, ApJ, 535, 464

Stein, R. F., \& Nordlund, A. 2001, ApJ, 546, 585

Straus, Th., Fleck, B., Severino, G., et al. 1998, ESA SP-417, 293

Strous, L. H., Goode, P. R., \& Rimmele, T. R. 2000, ApJ, 535, 1000

Warmuth, A., Hanslmeier, A., Messerotti, M., et al. 2000, Solar Phys., 194, 103

Wolff, C. L. 1972, ApJ, 176, 833 